\title{
Isoflurane increases tolerance to renal ischemia reperfusion injury compared to propofol

\author{
- an experimental study in pigs
}




\section{Abstract}

Purpose: To compare two clinically relevant anaesthetic agents, i.e. isoflurane versus propofol with respect to protection of the kidney in a porcine renal ischemia reperfusion model.

Materials and Methods: 14 hybrid pigs were randomized to anaesthesia with either isoflurane or propofol prior to laparoscopic surgery. Following anaesthesia, the left kidney hilum was clamped for 60 minutes and the right kidney removed. After 48 hours of reperfusion, urine was sampled for analysis of neutrophil gelatinase-associated lipocalin (NGAL), albumin and creatinine. The left kidney was harvested for histologic scoring of injury.

Results: Histologic examination of renal injury revealed a statistically significant difference in favour of isoflurane on denuded basement membrane score (isoflurane group $1.58 \pm 0.38$ vs. propofol 2.42 $\pm 0.80, \mathrm{p}=0.026)$. Median (25-75 percentile) urinary albumin $3.4 \mathrm{~g} / \mathrm{l}(2.25-7.48)$ vs. $8.9 \mathrm{~g} / \mathrm{l}(3.73-$ 13.8), ( $p=0.041)$ and urinary albumin/creatinine ratio $1.17(0.76-1.82)$ vs. $1.76(1.63-5.99),(p=$ 0.026) were both significantly lower in the isoflurane group. Median (25-75 percentile) urinary NGAL was 167 (51-215) pg/ml in the isoflurane group compared with 362 (149-508) pg/ml in the propofol group $(p=0.093)$.

Conclusion: Isoflurane increases tolerance to renal ischemia reperfusion injury compared to propofol in this model.

Key words: Ischemia-Reperfusion, Isoflurane, Kidney, Laparoscopy, Propofol. 


\section{Introduction}

Renal ischemia reperfusion injury (IRI) is a major concern in patients subjected to kidney transplantation or nephron sparing surgery. Depending on the complexity of the surgical procedure, the length of the window of renal ischemia may differ considerably. However, warm ischemia of more than 30 minutes may lead to irreversible renal injury with loss of nephrons and ultimately increases the risk of morbidity and mortality ${ }^{1,2}$. A common feature of kidney transplantation and nephron sparing surgery is the dependency on anaesthesia, for the procedure to be completed. Several studies have indicated that volatile anaesthetics may possess a protective effect against renal IRI in animal experimental studies with rodents ${ }^{3-8}$. Annecke et al have shown that sevoflurane prevented hemodynamic deterioration after reperfusion occlusion of the thoracic aorta for 90 minutes compared with propofol in a porcine model ${ }^{9}$. They also demonstrated a protection of the endothelium in sevoflurane treated pigs, which was not found in pigs anesthetized with propofol only ${ }^{10}$. Another study performed in rabbits demonstrated that the renal protection induced by desflurane was more effective than that of ischemic preconditioning ${ }^{11}$. Several studies on coronary artery bypass grafting patients and the impact of volatile anaesthetics in preventing postoperative acute kidney injury have also been published, although these results have been somewhat contradictive ${ }^{12-14}$. Propofol, an intravenously administered sedative and hypnotic agent frequently utilized for induction and maintenance of general anaesthesia, has also been shown to exhibit protection from renal IRI in rodents by reducing oxidative injury ${ }^{15-17}$. The hypothesis of the study was that either of these putative renoprotective anaesthetic agents, i.e. isoflurane or propofol respectively, would provide superior renoprotection in a porcine model with 60 minutes clamping of the renal vessels followed by 48 hours of reperfusion. 


\section{Materials and Methods}

The experimental protocol was approved by the local steering committee of the Norwegian Food Safety Authority. Animal care was done in accordance with the guidelines for the care and use of laboratory animals published by the U.S National institutes of Health (NIH Publication No. 85-23, revised 2011$)^{18}$.

14 domestic hybrid pigs (Noroc: Norwegian landrace and Yorkshire hybrids) from a local supplier were acclimated in the animal research facility 10-14 days before the experiments. The pigs were a mix of castrated males and females with a weight of $45 \pm 5 \mathrm{~kg}$. They were fasted overnight with free access to water and block randomized into two different anaesthesia protocols; i.e. isoflurane or propofol. The animals were premedicated with $15 \mathrm{mg} / \mathrm{kg}$ ketamine, $2 \mathrm{mg} / \mathrm{kg}$ atropine and $1 \mathrm{mg} / \mathrm{kg}$ midazolam. General anaesthesia in the isoflurane group was induced with fentanyl in an ear vein in addition to $4 \%$ isoflurane on a ventilation mask. The induction was followed by continuous isoflurane $2 \%$, fentanyl $0.02 \mathrm{mg} / \mathrm{kg} / \mathrm{h}$ and midazolam $0.3 \mathrm{mg} / \mathrm{kg} / \mathrm{h}$. In the propofol group general anaesthesia was induced with propofol and a fentanyl bolus in an ear vein. Maintenance of anaesthesia was accomplished by continuous propofol $14 \mathrm{mg} / \mathrm{kg} / \mathrm{h}$, fentanyl 0.02 $\mathrm{mg} / \mathrm{kg} / \mathrm{h}$ and midazolam $0.3 \mathrm{mg} / \mathrm{kg} / \mathrm{h}$. The animals were intubated and mechanically ventilated using a volume-controlled ventilator that was adjusted according to capnography measurements and arterial blood gases. The circulating volume was maintained by $10 \mathrm{mg} / \mathrm{kg} / \mathrm{h}$ saline infusion. When the animals were fully sedated and intubated, a central venous catheter was introduced into the jugular vein and an arterial cannula was inserted in the superficial femoral artery for hemodynamic measurements. The core temperature was maintained between $37-39{ }^{\circ} \mathrm{C}$ using a thermal mattress.

\section{Experimental protocol}

An overview of the experimental protocol is provided in Figure 1. In full anaesthesia, access to the abdominal cavity was gained through open placement of laparoscopic trocars in the midline. After 
verifying that the first port was inside the abdominal cavity, pneumoperitoneum was accomplished with a pressure of $10 \mathrm{mmHg}$. A total of three $12 \mathrm{~mm}$ trocars and two $5 \mathrm{~mm}$ trocars were inserted under visual guidance. The left renal hilum was mobilised and cleared. $2500 \mathrm{IU}$ heparin was administered intravenously 3 min before clamping. A Satinzky clamp was then placed over the hilum, artery and vein, and then clamped for $60 \mathrm{~min}$. During the clamping period, the right kidney was removed laparoscopically. Artery, vein and ureter were secured with hem-o-lok clips. The kidney was removed through a small midline incision. After 60 minutes, the Satinzky clamp was opened and the reperfusion of the kidney was observed by visualizing the change in colour. Then all the instruments were removed, and the abdomen was closed. The pigs were extubated and kept alive for 48 hours. Blood was sampled before surgery, and after 24 and 48 hours of reperfusion respectively (Figure 1). After 48 hours of reperfusion the pigs were sedated in the same way as previously described and euthanized with intravenous injection of $20 \mathrm{mg} / \mathrm{kg}$ sodium pentobarbital. The left kidney was then harvested for histological examination. Urine samples were collected directly from the bladder with a syringe.

\section{Histology and scoring of renal injury}

The kidney was fixed in formalin and sections from the upper, middle and lower part of the kidney were paraffin embedded. Multiple 4 micrometre sections were cut with a Micron HM 355S microtome and stained with haematoxylin/eosin and periodic acid-Shiff. The assessment of histologic kidney injury was performed by two experienced uropathologists (ER, SA) that were blinded for the groups. Histologic kidney injury was scored as previously described by Goujon et al ${ }^{19}$. Each of the following changes was given a score from 0-5. 0: No changes, 1 : criteria detected within less than 10 $\%$ of kidney samples, 2: Criteria detected within $10 \%$ to $25 \%$ of kidney samples, 3: Criteria detected within $25 \%$ to $50 \%$ of kidney samples, 4: Criteria detected within $50 \%$ to $75 \%$ of kidney samples, 5 : Criteria detected within more than $75 \%$ of kidney samples. The changes that were scored were the 
following: Atypical cytoplasmic vacuolization, cell detachment, denuded basement membrane, intracellular edema, tubular dilatation and tubular necrosis.

Urinary neutrophil gelatinase-associated lipocalin (NGAL)

Neutrophil gelatinase-associated lipocalin (NGAL) was analysed in urine samples collected after ischemia and 48 hours of reperfusion, before the pigs were euthanized. The urine was collected directly from the bladder with a syringe. The urine samples were stored at $-80^{\circ} \mathrm{C}$. Concentration levels of NGAL were achieved using a pig specific NGAL ELISA kit (BioPorto Diagnostics, Denmark). Duplicate samples were added to precoated wells and performed following manufacturer's instructions. Analysis and calibration of concentrations were performed using a Perkin Elmer Wallac 1420 Multilabel Counter (Marshall Scientific, NH).

\section{Statistics}

All statistical analyses were performed with computerized software (IBM SPSS statistics 25, Chicago, IL). P-values were calculated with independent samples Mann-Whitney U Test. Data are presented as mean \pm standard deviation or median and 25-75 percentiles, unless otherwise described. A significance level of 0.05 was utilized.

\section{Results}

Two pigs were excluded from the study. One pig was excluded because of a hematoma around the kidney that was discovered at the time of euthanasia. Another pig was excluded due to failure to remove the right kidney during ischemia, leaving 6 remaining animals in each group. Baseline characteristics of the two groups are displayed in Table 1, and revealed no differences with respect to weight, mean arterial pressure or haemoglobin between the groups. 
The mean level of creatinine after 48 hours of ischemia-reperfusion was lower in the isoflurane group (494 $\mu \mathrm{mol} / \mathrm{L} \pm 179$ ) compared to the propofol group $(522 \mu \mathrm{mol} / \mathrm{L} \pm 225)$, but the difference was not statistically significant (Table 2). A similar trend was found with respect to urea, with mean urea $8.8 \mathrm{mmol} / \mathrm{L} \pm 4.8$ in the isoflurane group versus $10.1 \mathrm{mmol} / \mathrm{L} \pm 6.0$ in the propofol group (ns) following ischemia and 48 hours of reperfusion.

An example of the different histological injury patterns scored by the pathologists are provided in Figure $2 A$ and $B$ and the results of the scoring of the two groups are demonstrated in Table 3. Overall, the mean histologic kidney injury score showed a difference in favour of isoflurane with a score of $1.85 \pm 0.48$ vs. $2.42 \pm 0.91$ in the propofol group, but the difference was not statistically significant $(p=0.39)$. However, a statistically significant difference in favour of isoflurane was found on denuded basement membrane score (Isoflurane group: $1.58 \pm 0.38$ versus propofol group $2.42 \pm 0.80, p=0.026$ ), indicating less renal injury in the isoflurane group compared to the propofol group.

The results from the urinary analysis are summarized in Figure $3(A-C)$. Median urinary albumin ( $25-75$ percentile) was $3.4 \mathrm{~g} / \mathrm{l}(2.25-7.48)$ in the isoflurane group compared to $8.9 \mathrm{~g} / \mathrm{l}$ (3.73 - 13.8) in the propofol group ( $p=0.041)$. Significantly lower levels of urinary albumin/creatinine ratio (ACR) were also found in the isoflurane group, with median ACR (25-75 percentile) 1.17 (0.76-1.82) in the isoflurane group compared to $1.76(1.63-5.99)$ in the propofol group $(p=0.026)$. Median $(25-$ 75 percentile) urinary NGAL was $167(51-215) \mathrm{pg} / \mathrm{ml}$ in the isoflurane group compared with 362 (149508) $\mathrm{pg} / \mathrm{ml}$ in the propofol group $(\mathrm{p}=0.093)$.

\section{Discussion}

Assessment of acute kidney injury has been a subject of controversy and discussion for decades, ${ }^{20}$ with histologic verified injury often referred to as the gold standard ${ }^{21}$. However, this method of evaluating renal injury is too invasive for widespread clinical use and has been criticized for not being sensitive enough when injury is subtle ${ }^{21}$. The methodological problem with low sensitivity for 
detection of minor injury on histologic evaluation is evident in the present study, with only a few animals displaying injury scores above the $10-25 \%$ criteria, despite 60 minutes of ischemia and a sixfold rise from baseline in both creatinine and urea. To strengthen our results, urinary biomarkers of acute kidney injury were utilized. Both urinary albumin and urinary ACR has been validated as biomarkers of acute kidney injury ${ }^{22}$. NGAL has been shown to be released into the urine from tubule cells as a response to ischemia reperfusion injury and has been extensively recognized as a biomarker in the same setting ${ }^{23}$.

The main finding in this study is the significantly lower denuded basement membrane score in the isoflurane group compared to the propofol group, indicating a reno-protective effect of isoflurane in this model. There were no differences with respect to atypical cytoplasmic vacuolization, cellular edema and cell detachment between the groups. However, these cellular changes are early and typically reversible manifestations of cellular injury ${ }^{2425}$. Since the findings of denuded basement membranes essentially is an indirect sign of clusters of dead cells, it may be argued that it represents the most significant manifestation of cellular injury among the six morphological changes measured in the Goujon scoring system ${ }^{19}$, and hence a strong argument for a protective effect of isoflurane over propofol.

The suggested reno-protective effect of isoflurane in this model was further strengthened by urinary analysis, demonstrating lower levels of urinary albumin as well as urinary ACR in the isoflurane group. Urinary NGAL was also found to be lower in the isoflurane group compared to the propofol group. However, the difference between the groups with respect to NGAL failed to reach statistical significance, possibly as a result of the limited number of animals in each group.

The single kidney porcine model presented here has been described previously in several other papers ${ }^{26-30}$. The reason to create a single kidney model is to avoid interference from the other kidney on the creatinine and urea levels. In the present model, some adjustments have been made. First, the right kidney was removed during the clamping of the left kidney, not before as done in previous studies ${ }^{26-30}$. The reason for this was to exclude any remote ischemic preconditioning that 
could have affected the results. All the surgery was done laparoscopically to minimise the stress on the animal and to achieve a closer resemblance to the clinical setting, with warm IRI during laparoscopic or robotic partial nephrectomy being the most obvious example. The background for harvesting the kidneys after 48 hours of reperfusion was based on the works by Jablonski et al ${ }^{31}$, who indicated this as an ideal time frame to detect the most extensive morphological changes on histologically examination, as opposed to later, when the kidney had already begun to repair and regenerate. In previous studies with this model the registered peak in creatinine levels was also measured after 48 hours. Gofrit et al has previously proposed serum creatinine as a surrogate marker for the glomerular filtration rate (GFR) in a single kidney model ${ }^{32}$. Although serum creatinine may very well reflect GFR in this model, the association between histologically based renal injury assessment and creatinine values was found to be poor by Jablonski et $a l^{31}$. The experience from our study is similar, demonstrating a poor correlation between creatinine levels and histologic markers of cellular injury. The substantial increase in creatinine 48 hours after ischemia was accompanied by only a modest injury to the renal cells on histological examination. The discrepancy between the considerable loss of renal function and the modest findings of cellular injury highlights the need for better markers to measure acute renal injury.

One of the intentions with the study was to develop an experimental model with a close resemblance to clinical practice. As previously mentioned, the laparoscopic approach utilized in the present study is similar to that of laparoscopic or robotic partial nephrectomy. There are important and obvious differences that must be considered before the results from these animal experiments can be transferred to the clinical setting with patients scheduled for nephron sparing surgery or a renal transplant. Firstly, the pigs used in these experiments are young and healthy, aged between three and four months and exhibiting a rapid growth pattern. Although kidney transplants from deceased donors may occur at any age, the majority of these kidneys are from donors older than 35 , whereas patients scheduled for laparoscopic partial nephrectomy are typically in their sixties and frequently suffer from atherosclerosis, diabetes and overweight. Secondly, the ischemic window in 
the present study of 60 minutes is considerably longer than what is expected of warm ischemia during partial nephrectomy. On the other hand, the ischemic trauma is probably better tolerated in young pigs than in older humans ${ }^{24}$. Some authors have demonstrated recovery of the porcine kidney after 90 minutes of warm ischemia ${ }^{2627}$. The optimal length of warm ischemia in this model may certainly be questioned, especially since the histological renal scoring only revealed a modest injury pattern. We may therefore speculate whether we would have achieved more rigorous differences between the groups if warm ischemia time had been increased to 75 or 90 minutes. We decided to use a window of 60 minutes of warm ischemia, since this previously has been described as the ideal length of ischemia to study renal reperfusion injury ${ }^{31}$. In our experience, 90 minutes of warm ischemia in this single kidney model increases mortality substantially, making it difficult to complete such an experimental protocol.

The comprehensive mechanisms involved in the reno-protection afforded by isoflurane are only partly understood. Briefly, isoflurane has been shown to influence multiple cytoprotective signaling pathways, including tumor growth factor $\beta 1$ (TGF- $\beta 1$ ) signaling, sphingosine kinase activation, adenosine receptor activation and IL-11 synthesis ${ }^{33}$. The isoflurane-mediated release of TGF- $\beta 1$ leads to CD73 synthesis, increasing renal tubular adenosine generation which ultimately protects against necrosis, apoptosis and inflammation through adenosine receptors $A_{1}$ and $A_{2 a}$. $A$ beneficial effect by isoflurane on the post-ischemic no-reflow phenomenom in acute kidney injury has also been suggested ${ }^{33}$. However, to elucidate the exact underlying mechanisms of how isoflurane exerts its reno-protective effect in this particular study, was beyond the scope of this work.

The cost and time of large animal experiments limits the sample size in experimental studies in general. It may be questioned whether this study was underpowered in the first place to be able to draw convincing conclusions. On the other hand, the findings of statistically significant differences with respect to denuded basement scoring, urinary albumin and urinary albumin/creatinine ratio between the groups remain a strong argument for the superiority of isoflurane over propofol in this 
model. However, more research is required to assess whether volatile anaesthetics such as isoflurane should be utilized for reno-protective purposes also, and not strictly for induction or maintenance of anaesthesia when protection of the kidney is critical.

\section{Acknowledgments:}

This work was supported by the Northern Norway Regional Health Authority under grant SFP112813.

\section{Disclosure:}

The authors report no conflict of interest. 


\section{Table 1.}

Baseline characteristics in pigs undergoing right sided nefrectomy and left sided clamping of the renal vessels for 60 minutes anaesthetized with either isoflurane or propofol.

\begin{tabular}{lccc}
\hline Baseline characteristics & $\begin{array}{c}\text { Isoflurane } \\
(\mathbf{n}=6)\end{array}$ & $\begin{array}{c}\text { Propofol } \\
(\mathbf{n}=6)\end{array}$ & $\boldsymbol{P}$ \\
\hline Weight $(\mathrm{Kg})$ & $46.3 \pm 5.0$ & $47.8 \pm 3.8$ & 0.6 \\
\hline Male/female & $2 / 4$ & $2 / 4$ & - \\
\hline $\mathrm{MAP}(\mathrm{mm} \mathrm{Hg})$ & $74.2 \pm 8.6$ & $84.4 \pm 13.9$ & 0.2 \\
\hline $\mathrm{Hb}(\mathrm{g} / \mathrm{dL})$ & $9.2 \pm 0.6$ & $9.7 \pm 0.8$ & 0.2 \\
\hline
\end{tabular}

MAP = Mean arterial pressure 


\section{Table 2.}

Creatinine and urea at baseline and following 24 and 48 hours of ischemia and reperfusion.

\begin{tabular}{lccc}
\hline & Isoflurane $(\mathbf{n}=6)$ & Propofol $(\mathbf{n}=6)$ & $\boldsymbol{P}$ \\
\hline Creatinine, baseline $(\mu \mathrm{mol} / \mathrm{L})$ & $81 \pm 7$ & $82 \pm 4$ & 0.6 \\
\hline Creatinine, 24 hours $(\mu \mathrm{mol} / \mathrm{L})$ & $413 \pm 114$ & $430 \pm 103$ & 0.7 \\
\hline Creatinine 48 hours $(\mu \mathrm{mol} / \mathrm{L})$ & $494 \pm 179$ & $522 \pm 225$ & 0.8 \\
\hline Urea, baseline $(\mathrm{mmol} / \mathrm{L})$ & $1.7 \pm 0.6$ & $1.6 \pm 0.7$ & 0.6 \\
\hline Urea 24 hours $(\mathrm{mmol} / \mathrm{L})$ & $8.5 \pm 3.1$ & $9.2 \pm 3.6$ & 0.7 \\
\hline Urea 48 hours $(\mathrm{mmol} / \mathrm{L})$ & $8.8 \pm 4.8$ & $10.1 \pm 6.0$ & 0.7 \\
\hline
\end{tabular}




\section{Table 3.}

Histology score in the isoflurane and propofol group.

\begin{tabular}{lccc}
\hline Histology & Isoflurane $(\mathbf{n}=\mathbf{6})$ & Propofol $(\mathbf{n}=6)$ & $\boldsymbol{P}$ \\
\hline Atypical cytoplasm vacuolization & $2.0 \pm 1.0$ & $1.8 \pm 0.8$ & 0.70 \\
\hline Cell detachment & $2.0 \pm 0.6$ & $2.5 \pm 1.1$ & 0.49 \\
\hline Denuded basement membrane & $1.6 \pm 0.4$ & $2.4 \pm 0.8$ & $0.026^{*}$ \\
\hline Intracellular edema & $1.8 \pm 1.0$ & $1.8 \pm 0.5$ & 0.82 \\
\hline Tubular dilatation & $1.9 \pm 0.7$ & $2.5 \pm 0.9$ & 0.24 \\
\hline Tubular necrosis & $1.9 \pm 0.8$ & $2.3 \pm 1.2$ & 0.82 \\
\hline Mean score & $1.9 \pm 0.5$ & $2.4 \pm 0.9$ & 0.39 \\
\hline
\end{tabular}

${ }^{*} p<0.05$ 


\section{Figure 1.}

The pigs were block randomized into two different anaesthesia protocols; i.e. isoflurane or propofol. Left renal vessels were clamped for 60 minutes, during which right kidney was removed laparoscopically. After 60 minutes the clamp was removed, the pigs were extubated and kept alive for 48 hours. Blood was sampled before surgery, and after 24 and 48 hours of reperfusion respectively. After 48 hours the left kidney was then harvested for histologic examination and urine was collected for analysis. $\mathrm{h}=$ hours, $\min =$ minutes. 


\section{Figure 2.}

Histology after one hour of ishaemia and 48 hours of reperfusion demonstrating the different morphological changes scored by the pathologist. A (left); Severe kidney damage. B (right); light kidney damage. ACV; Atypical cytoplasmic vacuolization, CD; Cell detachment, DBM; Denatured basement membrane, IE; Intracellular edema, TN; Tubular necrosis, TD; Tubular dilatation. 


\section{Figure 3.}

Urinary albumine (A), urinary albumin/creatinine ratio (B) and urinary NGAL (C) after one hour of sichemia and 48 hours of reperfusion in the isoflurane and propofol group. Data are presented with dots representing individual data. The bars indicate median and range. ${ }^{*} p<0.05$. 


\section{REFERENCES}

1. Patel AR, Eggener SE. Warm ischemia less than 30 minutes is not necessarily safe during partial nephrectomy: every minute matters. Urol Oncol 2011;29(6):826-8. doi:

10.1016/j.urolonc.2011.02.015 [published Online First: 2011/11/15]

2. Volpe A, Blute ML, Ficarra V, et al. Renal Ischemia and Function After Partial Nephrectomy: A Collaborative Review of the Literature. Eur Urol 2015;68(1):61-74. doi: 10.1016/j.eururo.2015.01.025 [published Online First: 2015/02/24]

3. Zhang $\mathrm{L}$, Huang $\mathrm{H}$, Cheng J, et al. Pre-treatment with isoflurane ameliorates renal ischemicreperfusion injury in mice. Life sciences 2011;88(25-26):1102-7. doi: 10.1016/j.Ifs.2011.04.004 [published Online First: 2011/05/14]

4. Hashiguchi $\mathrm{H}$, Morooka $\mathrm{H}$, Miyoshi $\mathrm{H}$, et al. Isoflurane protects renal function against ischemia and reperfusion through inhibition of protein kinases, JNK and ERK. Anesthesia and analgesia 2005;101(6):1584-9. doi: 10.1213/01.ane.0000184044.51749.b8 [published Online First: 2005/11/23]

5. Lee HT, Ota-Setlik A, Fu Y, et al. Differential protective effects of volatile anesthetics against renal ischemia-reperfusion injury in vivo. Anesthesiology 2004;101(6):1313-24. [published Online First: 2004/11/27]

6. Su MW, Chang SS, Chen $\mathrm{CH}$, et al. Preconditioning renoprotective effect of isoflurane in a rat model of virtual renal transplant. The Journal of surgical research 2014;189(1):135-42. doi: 10.1016/j.jss.2014.02.035 [published Online First: 2014/03/29]

7. Qin Z, Lv E, Zhan L, et al. Intravenous pretreatment with emulsified isoflurane preconditioning protects kidneys against ischemia/reperfusion injury in rats. BMC anesthesiology 2014;14:28. doi: 10.1186/1471-2253-14-28 [published Online First: 2014/04/18]

8. Liang Y, Li Z, Mo N, et al. Isoflurane Preconditioning Ameliorates Renal Ischemia-Reperfusion Injury through Antiinflammatory and Antiapoptotic Actions in Rats. Biological \& pharmaceutical bulletin 2014;37(10):1599-605. [published Online First: 2014/08/05]

9. Annecke T, Kubitz JC, Kahr S, et al. Effects of sevoflurane and propofol on ischaemia-reperfusion injury after thoracic-aortic occlusion in pigs. British journal of anaesthesia 2007;98(5):581-90. doi: 10.1093/bja/aem049 [published Online First: 2007/03/21]

10. Annecke T, Rehm M, Bruegger $D$, et al. Ischemia-reperfusion-induced unmeasured anion generation and glycocalyx shedding: sevoflurane versus propofol anesthesia. Journal of investigative surgery : the official journal of the Academy of Surgical Research 2012;25(3):162-8. doi: 10.3109/08941939.2011.618524 [published Online First: 2012/05/16]

11. Guye ML, Mc Gregor B, Weil G, et al. [Ischaemic and pharmacologic preconditioning: desflurane reduces renal reperfusion injury in rabbits]. Annales francaises d'anesthesie et de reanimation 2010;29(7-8):518-23. doi: 10.1016/j.annfar.2010.03.027 [published Online First: 2010/07/14]

12. Swyers T, Redford D, Larson DF. Volatile anesthetic-induced preconditioning. Perfusion 2014;29(1):10-5. doi: 10.1177/0267659113503975 [published Online First: 2013/09/05]

13. Sindhvananda W, Phisaiphun K, Prapongsena P. No renal protection from volatile-anesthetic preconditioning in open heart surgery. Journal of anesthesia 2013;27(1):48-55. doi: 10.1007/s00540012-1461-z [published Online First: 2012/08/14] 
14. Julier K, da Silva R, Garcia C, et al. Preconditioning by sevoflurane decreases biochemical markers for myocardial and renal dysfunction in coronary artery bypass graft surgery: a double-blinded, placebo-controlled, multicenter study. Anesthesiology 2003;98(6):1315-27. [published Online First: 2003/05/27]

15. Yuzbasioglu MF, Aykas A, Kurutas EB, et al. Protective effects of propofol against ischemia/reperfusion injury in rat kidneys. Renal failure 2010;32(5):578-83. doi: 10.3109/08860220903548940 [published Online First: 2010/05/22]

16. Yang S, Chou WP, Pei L. Effects of propofol on renal ischemia/reperfusion injury in rats. Experimental and therapeutic medicine 2013;6(5):1177-83. doi: 10.3892/etm.2013.1305 [published Online First: 2013/11/14]

17. Yang SC, Chung PJ, Ho CM, et al. Propofol inhibits superoxide production, elastase release, and chemotaxis in formyl peptide-activated human neutrophils by blocking formyl peptide receptor 1 . Journal of immunology (Baltimore, Md : 1950) 2013;190(12):6511-9. doi: 10.4049/jimmunol.1202215 [published Online First: 2013/05/15]

18. . In: th, ed. Guide for the Care and Use of Laboratory Animals. Washington (DC)2011.

19. Goujon JM, Hauet T, Menet E, et al. Histological evaluation of proximal tubule cell injury in isolated perfused pig kidneys exposed to cold ischemia. The Journal of surgical research 1999;82(2):228-33. doi: 10.1006/jsre.1998.5526 [published Online First: 1999/03/26]

20. Makris K, Spanou L. Acute Kidney Injury: Diagnostic Approaches and Controversies. Clin Biochem Rev 2016;37(4):153-75. [published Online First: 2017/02/09]

21. Waikar SS, Betensky RA, Emerson SC, et al. Imperfect gold standards for kidney injury biomarker evaluation. Journal of the American Society of Nephrology : JASN 2012;23(1):13-21. doi: 10.1681/ASN.2010111124 [published Online First: 2011/10/25]

22. Ware LB, Johnson AC, Zager RA. Renal cortical albumin gene induction and urinary albumin excretion in response to acute kidney injury. American journal of physiology Renal physiology 2011;300(3):F628-38. doi: 10.1152/ajprenal.00654.2010 [published Online First: 2010/12/15]

23. Mishra J, Ma Q, Prada A, et al. Identification of neutrophil gelatinase-associated lipocalin as a novel early urinary biomarker for ischemic renal injury. Journal of the American Society of Nephrology : JASN 2003;14(10):2534-43. [published Online First: 2003/09/30]

24. Schmitt R, Cantley LG. The impact of aging on kidney repair. 2008 doi: 10.1152/ajprenal.00543.2007

25. Sharfuddin AA, Molitoris BA. Pathophysiology of ischemic acute kidney injury. Nature reviews Nephrology 2011;7(4):189-200. doi: 10.1038/nrneph.2011.16 [published Online First: 2011/03/03]

26. Baldwin DD, Maynes $L$, Berger KA, et al. Laparoscopic warm renal ischemia in the solitary porcine kidney model. Urology 2004;64(3):592-7. doi: 10.1016/j.urology.2004.04.019 [published Online First: 2004/09/08]

27. Laven BA, Orvieto MA, Chuang MS, et al. Renal tolerance to prolonged warm ischemia time in a laparoscopic versus open surgery porcine model. The Journal of urology 2004;172(6 Pt 1):2471-4. 
28. Lyon MB, Orvieto MA, Zorn KC, et al. Effect of renal ischemia in laparoscopic acute versus chronic solitary kidney model. Urology 2007;69(2):402-6. doi: 10.1016/j.urology.2006.12.009

29. Orvieto MA, Zorn KC, Mendiola FP, et al. Ischemia preconditioning does not confer resilience to warm ischemia in a solitary porcine kidney model. Urology 2007;69(5):984-7. doi:

10.1016/j.urology.2007.01.100 [published Online First: 2007/05/08]

30. Orvieto MA, Zorn KC, Mendiola F, et al. Recovery of renal function after complete renal hilar versus artery alone clamping during open and laparoscopic surgery. J Urol 2007;177(6):2371-4. doi: 10.1016/j.juro.2007.01.115 [published Online First: 2007/05/19]

31. Jablonski P, Howden BO, Rae DA, et al. An experimental model for assessment of renal recovery from warm ischemia. Transplantation 1983;35(3):198-204. [published Online First: 1983/03/01]

32. Gofrit ON, Orvieto MA, Zorn KC, et al. Serum creatinine can be used as a surrogate for glomerular filtration rate in single renal unit models. The Canadian journal of urology 2009;16(1):4452-7; discussion 57.

33. Fukazawa K, Lee HT. Volatile anesthetics and AKI: risks, mechanisms, and a potential therapeutic window. Journal of the American Society of Nephrology : JASN 2014;25(5):884-92. doi:

10.1681/ASN.2013111215 [published Online First: 2014/02/11] 